\title{
DIGITAL TRANSFORMATION OF BUSINESS
}

\section{K. Schwertner}

\author{
Faculty of Economics and Business Administration, Sofia University, Bulgaria
}

\begin{abstract}
The paper presents opportunities of digital transformation of business as a changes associated with the application of digital technology in all aspects of business. A research of digital business found that maturing digital businesses are focused on integrating digital technologies, such as social, mobile, analytics/big data and cloud, in the service of transforming how businesses work. The ability to digitally reimagine the business is determined in large part by a clear digital strategy supported by leaders who foster a culture able to change and invent the new. Unique to digital transformation is that risk taking is becoming a cultural norm as more digitally advanced companies seek new levels of competitive advantage.

Among companies where big data, cloud, mobile, and social technologies are critical parts of the infrastructure, these technologies are, or will soon be profitable on average, had higher revenues, and achieved a bigger market valuation than competitors without a strong vision.

As with any emerging technology, however, there are significant challenges associated with cloud, mobile, social, and big data initiatives. The survey suggests that the primary risks preventing their wider adoption are data security issues, lack of interoperability with existing IT systems, and lack of control.
\end{abstract}

Key words: cloud, mobile, social technologies, big data, analytics, internet of things, change management

\section{INTRODUCTION}

Digital Business Transformation is disrupting businesses in every industry by breaking down barriers between people, businesses and things. By breaking these barriers, they are able to create new products, services and find more efficient ways of doing business. These innovations are happening across organizations of all types, in every industry. But they share a common theme: The ability to transform processes \& business models, Empower workforce efficiency and innovation, and Personalize customer/ citizen experiences. To do this, companies need a Digital Business Platform that is outcome driven and enabled by technology (2).

Digital Business Transformation is the application of technology to build new business models, processes, software and systems that results in more profitable revenue, greater competitive advantage, and higher efficiency. Businesses achieve this by transforming processes and business models, empowering workforce efficiency and innovation, and personalizing customer/citizen experiences.

Among companies where big data, cloud, mobile, and social technologies are critical parts of the infrastructure, these technologies are, or will soon be profitable on average, had higher revenues, and achieved a bigger market valuation than competitors without a strong vision.

As with any emerging technology, however, there are significant challenges associated with cloud, mobile, social, and big data initiatives. The survey suggests that the primary risks preventing their wider adoption are data security issues, lack of interoperability with existing IT systems, and lack of control.

The paper research an organization's level of digital maturity and readiness for digital business transformation. Digital business transformation is a new phenomenon, and no organization can be considered yet to have achieved the end state of maturity.

\section{STRATEGY IS BEFORE TECHNOLOGY}

Digital technologies - social, mobile, analytics and cloud - are impacting organizations and most areas of human activity. Organizations need to integrate these digital technologies and their capabilities to transform processes, engage talent and drive new business models to compete and strive in the digital world.

Organizational change occurs when a company makes a transition from its current state to some desired future state. Managing organizational change is the process of planning and implementing change in organizations in such a 
SCHWERTNER $K$.

way as to minimize employee resistance and cost to the organization while simultaneously maximizing the effectiveness of the change effort.

Digital business transformation is the integration of new digital technologies into all business areas, leading to a fundamental change in the way the organization works.

In digital transformation, it is not enough to use as many technologies as possible. The strategy is moving, it must have a clear vision for the company's development, and then be supported by the unlimited possibilities of these technologies that are related to the chosen strategy.

Successful digital transformation goes hand in hand with reengineering and optimization of business processes in the most appropriate way for the strategy. The digital transformation of the business seems different for different companies and it is difficult to give a strategy that is valid for everyone.

Only one platform is needed to connect all business units in the organization. Without a single platform, digital transformation can't be achieved. The goal should be a seamless interaction with the customer at all touch points of business.

According to a study by the Massachusetts Institute of Technology, digital-transformed businesses are 26 percent more profitable than norms.

Without the necessary strategy, too many companies are focused on technology rather than on the customer. It is necessary that organizational change, technology and data integration are addressed equally to achieve successful digital transformation of business.

Successful organizations must leverage strategy, culture and leadership to harness the potential of digital transformation of business. Organization's digital strategy goal are: improve customer experience, increase efficiency, improve innovation, improve decision making, transform the business. The organization is innovative is innovative compared to their competitors. Leadership has sufficient skills, experience to lead digital strategy.

Digital business transformation can only be successful if there is a well-founded strategy and leadership. Transformational changes are required to implement the digital transformation, which is related to strategy, leadership and organizational culture. Business practice research has shown that businesses with a successful digital-based business have a clear strategy, commitment to senior management with change, motivated employee involvement in the process, and focusing on changes to customer needs and interests.

One of the approaches to exploring digital transformation is the grouping of changes in three areas: consumer behavior, business processes and business models (3).

The transformation of consumer experiences in the use of products and services of the organization is expressed in the in-depth study of market segments and their behavior in the marketing space, consumer behavior and loyalty, interactive communication with customers in the sales process and many digital contact points between the organization and the customers.

Transformation of the business processes of the organization covers the automation of $\mathrm{R} \& \mathrm{D}$, production and distribution processes. Digital technologies also enable the capacity of people to work at different levels in different functional areas. Increasing distance work at the employees' home, decision-making on the basis of real customer relationship data helps to speed up decision-making on the availability of production in different production units.

Transforming the business model is done through digital business modification, a new digital business and digital globalization. These processes take place by adding digital content to existing products and services and introducing new digital solutions.

Characteristic of modern man is his digital connectivity, mobility, existence as part of a larger digital community. These features, related to the business segment element of a business model, mean the digital business segmentation and customer relationship solutions that have specific information requirements (Data), global customer access (Cloud Services) Grouping customer purchases, and more according to SAP White Paper Digital Transformation (6).

Digital business transformation is seen as a set of 7 elements: business model, organizational structure, digital skills of employees, digitization of business processes, IT infrastructure, digitization of products / services, digital channels for interaction with clients (3).

Rapid changes in the digital environment entail shortening strategic planning times and shrinking to annual planning, careful handling of extrapolation of existing data, perception of the need for continuous change, decentralized management, etc. 


\section{DIGITAL TRANSFORMATION MANUFACTURING COMPANIES}

In some sectors, digital transformation primarily involves production processes. This is particularly typical for manufacturing companies. Reducing costs by digitizing the processes of developing, testing and producing new products is of paramount importance. Mobile applications are more important to improving the production processes and internal communications of employees than to interact with customers who are mostly not end-users. Large databases and information processing are more focused on production (1). The digitization of production processes opens up many opportunities for expanding business and for its internationalization / globalization.

The digitization of production processes, however, opens up many opportunities for the expansion of business and for its internationalization in traditional economic sectors.

The industry's traditional value chain of original equipment manufacturers (OEMs), suppliers, retailers and the aftermarket has been disrupted by new, digitally astute entrants in both the existing and extended value chain. New technologies have propelled business model innovations that have challenged and extended the standard value chain in offering new products and services to the consumer (8).

The digital trends have led to a growing relevance of new entrants in traditional segments and creation of new segments.

The speed of this transformation is governed by the advances in connectivity technology, changes in consumer behavior, the emergence of new business models, and by environmental trends and regulatory practices. The impact has been seen mostly in the aftersales stage of the value chain. However, digital is also having a significant transformational impact on R\&D, procurement, assembly, marketing, and sales. In the parts segment, 10 to $15 \%$ of all global revenue will be generated online by 2025, and for parts and service retailing, China will be the most attractive market for revenue growth in digitization.

Players in the industry must also respond to fundamental consumer expectations around security and data privacy. The ability to manage and secure consumer data is a challenge faced by most industries in this increasingly digital world.

Digital transformation requires an in-depth analysis of the current state of business processes and business model in the organization. The analysis precedes the development of a digital transformation strategy. The analysis should answer questions grouped into several areas: Digital Transformation Attitude: Managing Support for Digital Business Strategy; The degree of use of digital technologies in the work of staff; The degree of use of digital communication channels; Digital infrastructure; Digital tools that meet customer needs and internal processes; Investments in digital solutions - what resources can be allocated to processes of digital transformation.

The analysis should focus on a number of key areas: users, suppliers and partners, investors, organization staff and organization leadership. The analysis should show how an organization's digitization will create more value for consumers, how it will help investor relations, how it will enhance interaction with partners, how it will change corporate culture, and how effectively the change in organization and process to digital transformation (7).

Digital transformation also includes the provision of services of the same high quality through all access channels at any time using cloud services and mobile applications.

\section{TECHNOLOGY ASPECTS OF DEGITAL BUSINESS TRANSFORMATION}

Cloud computing is a model for enabling convenient, on-demand network access to a shared pool of configurable computing resources (e.g., networks, servers, storage, applications, and services) that can be rapidly provisioned and released with minimal management effort or service provider interaction. This cloud model promotes availability and is composed of five essential characteristics (On-demand self-service, Broad network access, Resource pooling, Rapid elasticity, Measured Service); three service models (Cloud Software as a Service (SaaS), Cloud Platform as a Service (PaaS), Cloud Infrastructure as a Service (IaaS)); and, four deployment models (Private cloud, Community cloud, Public cloud, Hybrid cloud). Key enabling technologies include: (a) fast wide-area networks, (b) powerful, inexpensive server computers, and (c) high-performance virtualization for commodity hardware (4).

Cloud computing is a new technology for the enterprises. Companies in all vertical markets and company sizes will increasingly rely on public cloud services. Some differences will however apply. Large enterprises (over 250 employees), who already represent more than $80 \%$ of current cloud spending, will continue investing more than small and middle enterprises (SMEs). Among SMEs, larger ones (with 100-249 employees) are expected to increase spending faster than smaller 
ones. Tangible economic benefits are available from the adoption of cloud in the EU amongst enterprises.

The most important advantages of cloud computing are a reduction of expenses, technical staff and efforts.

Business benefits from cloud computing:

- Predictable costs - cloud services are typically paid on a monthly basis or based on use with little or no upfront costs. This means that instead of making a substantial initial capital investment, technology or services are purchased by existing operating costs.

- Reduced total cost - benefits of cloud services derived from economies of scale that a service provider can achieve.

- Access to the best technology - cloud services enable organizations to benefit from the best technologies, without making any initial costs.

- Charging in use - in the model of cloud services, organizations pay for actual rather than maximum use.

Adoption of cloud is already widespread and still growing in the EU in both the private and public sectors.

- For clients that have used cloud, the attraction for further adoption relates to efficiencies, agility and flexibility - for those that have not yet started lower cost and ease of use are the main attractions.

- Business benefits included more effective mobile working (46\%), higher productivity $(41 \%)$, more use of standard processes $(35 \%)$, better ability to enter new business areas (33\%) and the ability to open up in new locations $(32 \%)$.

Over the last few years, cloud technologies have been developing extremely rapidly, and forecasts show that this trend will continue as they provide an instrument for optimal resource efficiency. Focus will continue on security and service management.

Data show that $67 \%$ of small and medium-sized businesses in Bulgaria currently have some type of cloud services, while the average for Europe is $53 \%$.

Business predictions are that by 2025 all ICT business solutions will be realized in a cloud, which means they will use cloud technology and cloud models. More than $85 \%$ of corporate applications will be located directly in the cloud.

The Internet of things (IoT) is the internetworking of physical devices, vehicles (also referred to as "connected devices" and "smart devices"), buildings, and other items - embedded with electronics, software, sensors, actuators, and network connectivity that enable these objects to collect and exchange data. In 2013 the Global Standards Initiative on Internet of Things (IoTGSI) defined the IoT as "the infrastructure of the information society." The IoT allows objects to be sensed or controlled remotely across existing network infrastructure, creating opportunities for more direct integration of the physical world into computer-based systems, and resulting in improved efficiency, accuracy and economic benefit in addition to reduced human intervention (9). Cloud IoT service is a part of many successful business information systems.

Mobile Technology is important part of the digital transformation technologies.

- Mobile technologies realize the goal of digital transformation and provide seamless interaction with the customer at all touch points with business.

- The growth of a mobile digital business platform based on smartphones or tablets.

- Business transforming society and the global economy are work mobility and digital collaboration.

- The benefits of mobile technologies include greater productivity, 26 percent more profitable than normal.

The use of mobile technologies in business and the current level of integration between technologies entirely caused by the needs of the enterprise and focused on optimal business processes management.

Big Data and Data Analysis - The volume of business data (terabytes and increasingly petabytes of information) suggests why managing and analysing it is a challenge. It's no longer efficient for data warehouses (DWs) to manage single, homogenous workloads.

- Pooling data resources in a cloud model allows for greater flexibility and faster innovation for dynamic business demand. Cloud computing has changed the parameters that have enforced the traditional relational database restrictions because it delivers dynamic resource allocation, virtualization, and dramatic economies of scale for managing large amounts of data.

- Business Intelligence (BI) tools, Google BigQuery web service for big data and Google CloudSQL for relational database.

In 2016, about three-quarters of companies based in the European Union (EU) and a team of at least 10 people had a website and nearly half used social media. However, only $10 \%$ of them say they are also analyzing large data, according to Eurostat (European Statistical Office). 
The most popular sources of analysis are geo location data from portable devices used by $47 \%$ of businesses and data generated by social media $(45 \%)$. One third (33\%) reported that they analyzed their own large data generated by smart devices and sensors, and $25 \%$ used "other sources". Of the EU Member States for which such information is available, at least $15 \%$ of companies in the EU use the analysis of large data: Malta and the Netherlands (19\%), Belgium (17\%), Finland and the United Kingdom (15\%), Bulgaria $7 \%$. On the other hand, only $6 \%$ of companies analyze large data in Germany and Poland, and in Cyprus this percentage is $3 \%$.

\section{DIGITAL BUSINESS TRANSFORMATION IN SME}

Within the DIGITRANS project "Digital Transformation in the Danube Region", six Bulgarian SMEs (Small and Medium-sized Enterprises) have been contacted. The results of the interviews with Bulgarian SMEs show that they have all realized that the digitization of their business models is quite up to date, important and urgent. The topic of digitization and transformation of the business model is crucial for building a competitive advantage and preserving the competitiveness of the local and international market for all organizations (5).

All levels of the hierarchy, managers and owners are directly involved in the processes of digital transformation. This proves that SME managers are fully aware of the important role that digital business transformation performs.

The main difficulties and obstacles to the digitization of companies are not technologies, but human factors, cultural traditions, employees' resistance to change, lack of relevant knowledge and good practices, lack of adequate resources, lack of motivation and risk taking.

The 2016 annual International Data Corporation (ICT) forecast of the ICT industry focuses on the strong impact of digital transformation, an industrial platform for growth and innovation. Digital transformation unites mobile apps and devices, cloud services, large data analyzes (Big Data) and social networks. According to IDC's forecast, by 2020 , the market for information and communication technology will reach $\$ 5$ trillion. (Compared to $\$ 1.3$ trillion in 2012), with $40 \%$ of revenue and $98 \%$ of growth being provided by digital transformation technologies, which are now only $22 \%$ of IT spending.

\section{CONCLUSION}

Organizations today are looking for a good business environment that does not negatively affect corporate flexibility. One of the key success factors in the global business environment is for the company to be able to adapt quickly and effectively to changes.

Digital business transformation is an objective process that responds to changing business environments. Its implementation requires a clear strategy and prioritization supported by financial resources, leadership and active participation of all employees in the organization. The speed of flow depends on the specifics of the sector.

Rapid expansion in global connectivity is the current trend. Most dramatically, the world economy is transforming into a digital economy with a proliferation of cloud computing, big data and analytics, mobility and broadband connectivity, e-commerce, social media and the use of smart sensors and the Internet of Things.

Currently strategic technologies that can significantly affect the corporate market the next years, are formed under the influence of four convergent forces - social networks, mobile devices, cloud computing, and data analytics. These forces are innovative and revolutionary in themselves, but in combination, they radically transform business and society, destroying old business models and creating new leaders. Intersection of these powers built a foundation for digital transformation platforms.

\section{REFERENCES}

1. Bonnet, D., P. Ferraris, 2013. Digital Transformation Benchmark - 2012: Insights for the Manufacturing Industry

2. Bradley J., L. James, J. Macaulay, A. Noronha, M. Wade, 2015, Digital Vortex. How Digital Disruption Is Redefining Industries, Global Center for Digital Business Transformation, Available online

http://www.cisco.com/c/dam/en/us/ solutions/collateral/industry-solutions/digitalvortex-report.pdf

3. Kane, G., D. Palmer, A. Phillips, D. Kiron, N. Buckley, 2015. Strategy, Not Technology Drives Digital Transformation, MIT Sloan Management Review and Deloitte University Press, July 2015. Available online at http://sloanreview.mit.edu/ projects/strategydrives-digital-transformation/

4. NIST Cloud Computing Program - NCCP https://www.nist.gov/programs-projects/nistcloud-computing-program-nccp

5. Project DIGITRANS http://www.interreg-danube.eu/approvedprojects/digitrans

6. SAP White Paper Digital Transformation. Digital Business Modeling: A Structural Approach Toward Digital Transformation (2016) Available online at https://news.sap.com/digital-business- 
modeling-a-structural-approach-toward-digitaltransformation/

7. The digitization of everything - How organizations must adapt to changing consumer behavior, Ernst and Young, http://www.ey.com

8. World Economic Forum White Paper Digital Transformation of Industries: In collaboration with Accenture Digital Enterprise (2016), Available online

http://reports.weforum.org/digital-

transformation-of-industries/wp-content/blogs. $\mathrm{dir} / 94 / \mathrm{mp} /$ files/pages/files/digital-enterprise-

narrative-final-january-2016.pdf

9. https://en.wikipedia.org/wiki/Internet_of_thing 
SCHWERTNER K. 